\title{
Higher Order Statistics for Texture Analysis and Physical Interpretation of Polarimetric SAR Data
}

\author{
Xinping Deng, and Carlos López-Martínez, Senior Member, IEEE
}

\begin{abstract}
The logarithmic cumulants (log-cumulants for short) of the second and third order are widely used in the statistical analysis of PolSAR data. However, both the product model and the finite mixture model may produce the same values of these statistics, which means the use of these log-cumulants is not enough to determine the statistical model of the data. In this paper, it is demonstrated that the log-cumulants of higher orders can help to distinguish the concept of texture from that of mixture, providing a physical insight into the data statistics. A tool called log-cumulant cube, that helps to visualize this difference, is proposed by considering texture distributions from the Pearson's family. Results on both simulated and real SAR data show that the use of higher order statistics is useful when it comes to texture analysis of PolSAR data.
\end{abstract}

Index Terms-Polarimetric SAR, Texture Analysis, Log Cumulant, Higher Order Statistics.

\section{INTRODUCTION}

Knowledge of the exact statistical properties of the signal plays an important role in characterizing polarimetric synthetic aperture radar (PolSAR) data. Gaussian statistics for the radar return signals have been frequently assumed. However, the analysis of real PolSAR images reveals that non-Gaussian models give a better representation. In the last two decades, a considerable research effort has been dedicated to find accurate and efficient non-Gaussian models for PolSAR data, in the framework of the product model which assumes that the radar return is a product of two independent components, the texture and the speckle [1]-[5]. The product model is also referred to as scale mixture of Gaussian distributions [6]. Theoretically, the texture could be described by any distribution, but here we confine ourselves to the Pearson ones, since they are the most common and widely studied distributions to model texture, which could give explicit expressions for the distributions of the product model [5], [7]-[9].

Additionally, the heterogeneity that appears in PolSAR data may result from the mixture of different targets. For instance, from an urban area which usually consists of different objects like houses, trees and roads, the backscattering is a combination of different scattering mechanisms. The forest areas sometimes can be treated as a mixture of bright clutters and dark ones, corresponding to the strong returns from the crowns of trees and the shadows behind them. To represent this

Manuscript received XXX, XXXX; revised XXX, XXXX. This work has been financed by the Spanish Science, Research and Innovation Plan (MINECO) with Project Code TIN2014-55413-C2-1-P.

Xinping Deng and Carlos López-Martínez are with the Remote Sensing Laboratory, Signal Theory and Communications Department, Universitat Politècnica de Catalunya, 08034 Barcelona, Spain. E-mail: xinping.deng@upc.edu, carlos.lopez@tsc.upc.edu. type of data, a simple product model would be inappropriate. Finite mixture models, instead, could achieve reasonable level of accuracy [10]-[13].

The product model and the finite mixture model describe two quite different scattering scenarios, the former models a single type of target with textures, whereas the latter represents combinations of various targets. In a previous study [14], however, it is shown that the same log-cumulants of the second order and the third order [8], [9] may result from both of them. There is an ambiguity between the texture and mixture according to those statistics. As they have different physical meanings, it is necessary to discriminate the concept of texture from that of mixture. In this paper, we demonstrate that higher order statistics, and the fourth order log-cumulant in particular, are useful to accomplish this task. In this work, only texture distributions from the Pearson's family are considered.

\section{PolSAR Data Models}

\section{A. Product Model}

Under the assumption of the product model [1], the scattering vector obtained by a system can be written as

$$
\mathbf{k}=\sqrt{\tau} \mathbf{z} .
$$

Here $\tau$ is the texture parameter, a positive random variable with unity mean value, and $\mathbf{z}$ is the speckle vector, following a multivariate circular Gaussian distribution with zero mean and covariance matrix $\boldsymbol{\Sigma}=E\left\{\mathbf{z z}^{\dagger}\right\}$, where $E\{\cdot\}$ is the expectation operator and $(\cdot)^{\dagger}$ is the transpose conjugate operation.

The scattering vectors are frequently multilook processed and represented by the sample covariance matrices

$$
\mathbf{C}_{L}=\frac{1}{L} \sum_{i=1}^{L} \mathbf{k}_{i} \mathbf{k}_{i}^{\dagger}=\frac{\tau}{L} \sum_{i=1}^{L} \mathbf{z}_{i} \mathbf{z}_{i}^{\dagger}
$$

where $L$ is the number of looks. Here it is assumed that the texture variable is constant within the multilook cell [2]. Let $d$ denote the number of dimensions and $|\cdot|$ the matrix determinant, then the probability density function (PDF) is obtained by averaging all possible $\tau$

$$
\begin{aligned}
p\left(\mathbf{C}_{L} ; L, \boldsymbol{\Sigma}\right)= & \int_{0}^{\infty} \frac{L^{L d}\left|\mathbf{C}_{L}\right|^{L-d}}{\Gamma_{d}(L) \tau^{L d}|\mathbf{\Sigma}|^{L}} \\
& \times \exp \left(-\frac{L}{\tau} \operatorname{Tr}\left(\boldsymbol{\Sigma}^{-1} \mathbf{C}_{L}\right)\right) p(\tau) d \tau
\end{aligned}
$$

where $\operatorname{Tr}(\cdot)$ is the matrix trace operation and $\Gamma_{d}(L)$ is the generalized gamma function given by

$$
\Gamma_{d}(L)=\pi^{d(d-1) / 2} \prod_{i=1}^{d} \Gamma(L-i+1)
$$


with $\Gamma(\cdot)$ representing the gamma function.

A number of models have been proposed in the literature by assuming different distributions for the texture parameter $\tau$, including the Wishart distribution, the $\mathcal{K}$-distribution [2], the $\mathcal{G}^{0}$-distribution [3], the Kummer- $\mathcal{U}$ distribution [4], and the $\mathcal{W}$ and $\mathcal{M}$ distribution [5], see Table I. The texture distributions of all these models are from the Pearson's family, and they can give closed form expressions for the PDFs of the sample covariance matrix, as well as the main testing statistic, logcumulants.

\section{B. Finite Mixture Model}

Assume that the region under analysis can be modeled by a mixture of $K$ components, then the overall PDF of the data can be written as a weighted sum of the probabilities of each component [10], [13]

$$
p\left(\mathbf{C}_{L} ; \boldsymbol{\theta}\right)=\sum_{i=1}^{K} w_{i} p_{i}\left(\mathbf{C}_{L} ; \boldsymbol{\theta}_{i}\right)
$$

where $\boldsymbol{\theta}$ is a vector collecting all the parameters of the distribution and the mixing proportions obey

$$
\sum_{i=1}^{K} w_{i}=1, w_{i} \geq 0
$$

It has been shown that for complicated regions with more irregular histograms (multimodal, spiky), the finite mixture model is more accurate than a single distribution [10]-[12]. There are many options for the distributions of the mixing components, but here we mainly focus on the mixture of Wishart distributed components.

\section{Log-Cumulants}

To test if a model is suitable for the data, a measurable statistic is required. It is demonstrated that Mellin kind statistics are of great value for the analysis of the sample covariance matrix, and that they can be employed to determine the model fit [8], [9]. Define the Mellin kind matrix-variate characteristic function as the Mellin transform of the PDF [9]

$$
\phi(s)=\int_{\Omega_{+}}\left|\mathbf{C}_{L}\right|^{s-d} p\left(\mathbf{C}_{L}\right) \mathrm{d} \mathbf{C}_{L}
$$

then, the $v$ th order log-moment and log-cumulant are given by

$$
\mu_{v}=\left.\frac{d^{v}}{d s^{v}} \phi(s)\right|_{s=d}, \quad \kappa_{v}=\left.\frac{d^{v}}{d s^{v}} \ln \phi(s)\right|_{s=d} .
$$

They are related by the combinatorial version of Faà di Bruno's formula

$$
\begin{aligned}
\kappa_{v} & =g_{v}\left(\mu_{1}, \cdots, \mu_{v}\right) \\
& =\mu_{v}-\sum_{i=1}^{v-1}\left(\begin{array}{c}
v-1 \\
i-1
\end{array}\right) \kappa_{i} \mu_{v-i} .
\end{aligned}
$$

The sample log-cumulants can be estimated using $\hat{\kappa}_{v}=$ $g_{v}\left(\hat{\mu}_{1}, \cdots, \hat{\mu}_{v}\right)$ where $\hat{\mu}_{v}$ is the estimated log-moments

$$
\hat{\mu}_{v}=\frac{1}{N} \sum_{i=1}^{N}\left(\ln \left|\mathbf{C}_{i}\right|\right)^{v}
$$

with $N$ denoting the number of samples and $\mathbf{C}_{i}$ the $i$ th sample covariance matrix. As shown in [15], the variance of $\hat{\kappa}_{v}$ is given by

$$
\operatorname{Var}\left\{\hat{\kappa}_{v}\right\}=\frac{1}{N} \nabla g_{v}^{T} \mathbf{M} \nabla g_{v}
$$

where

$$
\nabla g_{v}=\left[\frac{\partial g_{v}\left(\mu_{1}, \cdots, \mu_{v}\right)}{\partial \mu_{1}}, \cdots, \frac{\partial g_{v}\left(\mu_{1}, \cdots, \mu_{v}\right)}{\partial \mu_{v}}\right]^{T}
$$

and $\mathbf{M}$ is the covariance matrix of log-moments with entries $\mathbf{M}_{i j}=\mu_{i+j}-\mu_{i} \mu_{j}$.

To see if a model is applicable, we can compare the logcumulants calculated from the PDF $\left(\kappa_{v}\right)$ and those estimated from the sample data $\left(\hat{\kappa}_{v}\right)$. In [9], a diagram is proposed to visualize the comparison by plotting the second order $\log$-cumulant $\kappa_{2}$ against the third order log-cumulants $\kappa_{3}$ in a plane, where different product models place in different regions. The distributions shown in Table I cover the whole log-cumulant diagram space [5].

\section{Log-Cumulants of the Finite Mixture Model}

Assume that the mixing components follow complex Wishart distributions such that the matrix-variate Mellin transform [9] of the finite mixture model in (5) can be written as

$$
\phi(s)=\left(\sum_{i=1}^{K} w_{i}\left|\boldsymbol{\Sigma}_{i}\right|^{s-d}\right) \frac{\Gamma_{d}(L+s-d)}{\Gamma_{d}(L)}
$$

where $\boldsymbol{\Sigma}_{i}$ is the covariance matrix of the $i$ th component, and $\Gamma_{d}(L)$ is the normalization factor given by (4). Let $|\boldsymbol{\Sigma}|_{\text {min }}$ denote the minimum determinant, $|\boldsymbol{\Sigma}|_{\text {min }}=\min \left(\left\{\left|\boldsymbol{\Sigma}_{i}\right|, i=\right.\right.$ $1, \cdots, K\})$, and $\rho_{i}=\left|\boldsymbol{\Sigma}_{i}\right| /|\boldsymbol{\Sigma}|_{\text {min }} \geq 1$, then the Mellin transform can be reformulated as

$$
\phi(s)=\left(\sum_{i=1}^{K} w_{i} \rho_{i}^{s-d}\right) \frac{|\boldsymbol{\Sigma}|_{\min }^{s-d} \Gamma_{d}(L+s-d)}{\Gamma_{d}(L)} .
$$

According to (8), the log-cumulants can be calculated as

$$
\begin{aligned}
& \kappa_{1}=\psi_{d}^{(0)}(L)+\beta_{1}+\ln |\boldsymbol{\Sigma}|_{\text {min }} \\
& \kappa_{v>1}=\psi_{d}^{(v-1)}(L)+\beta_{v}
\end{aligned}
$$

where $\psi_{d}^{(v-1)}(L)$ is the multivariate extension of the polygamma function due to the multilook, and $\beta_{v}$ is the result from the mixture which can be solved recursively by

$$
\beta_{v}=r_{v}-\sum_{k=1}^{v-1}\left(\begin{array}{l}
v-1 \\
k-1
\end{array}\right) \beta_{k} r_{v-k}
$$

with $r_{v}=\sum_{i=1}^{K} w_{i}\left(\ln \rho_{i}\right)^{v}$.

In the case of two mixing components, we have $r_{v}=$ $w_{1}\left(\ln \rho_{1}\right)^{v}+w_{2}\left(\ln \rho_{2}\right)^{v}$, and either $\rho_{1}$ or $\rho_{2}$ is equal to 1 according to their definition. Ignoring the subscript, $r_{v}$ is reduced to $w(\ln \rho)^{v}$, and the $\log$-cumulants of the first several orders are

$$
\begin{aligned}
& \kappa_{2}=\psi_{d}^{(1)}(L)+w(1-w)(\ln \rho)^{2} \\
& \kappa_{3}=\psi_{d}^{(2)}(L)+w(1-w)(1-2 w)(\ln \rho)^{3} \\
& \kappa_{4}=\psi_{d}^{(3)}(L)+w(1-w)\left(1-6 w+6 w^{2}\right)(\ln \rho)^{4} .
\end{aligned}
$$


TABLE I

Product Models AND Log-Cumulants

\begin{tabular}{|c|c|c|}
\hline Model & Texture Distribution & Log-Cumulants of $v$ th Order \\
\hline Wishart & $p(\tau)=\delta(\tau-1)$ & $\begin{array}{l}\kappa_{1}=\psi_{d}^{(0)}(L)+\ln |\boldsymbol{\Sigma}|-d \ln L=C \\
\kappa_{v>1}=\psi_{d}^{(v-1)}(L)\end{array}$ \\
\hline $\mathcal{K}$ & $p(\tau ; \alpha)=\frac{1}{\Gamma(\alpha)} \frac{(\alpha \tau)^{\alpha}}{\tau} \exp (-\alpha \tau)$ & $\begin{array}{l}\kappa_{1}=C+d\left(\psi^{(0)}(\alpha)-\ln \alpha\right) \\
\kappa_{v>1}=\psi_{d}^{(v-1)}(L)+d^{v} \psi^{(v-1)}(\alpha)\end{array}$ \\
\hline $\mathcal{G}^{0}$ & $p(\tau ; \lambda)=\frac{1}{\Gamma(\lambda)} \frac{(\lambda-1)^{\lambda}}{\tau^{\lambda+1}} \exp \left(-\frac{\lambda-1}{\tau}\right)$ & $\begin{array}{l}\kappa_{1}=C+d\left(-\psi^{(0)}(\lambda)+\ln (\lambda-1)\right) \\
\kappa_{v>1}=\psi_{d}^{(v-1)}(L)+d^{v}(-1)^{v} \psi^{(v-1)}(\lambda)\end{array}$ \\
\hline Kummer- $\mathcal{U}$ & $p(\tau ; \xi, \zeta)=\frac{\Gamma(\xi+\zeta)}{\Gamma(\xi) \Gamma(\zeta)} \frac{\xi}{\zeta-1}\left(\frac{\xi}{\zeta-1} \tau\right)^{\xi-1}\left(\frac{\xi}{\zeta-1} \tau+1\right)^{-\xi-\zeta}$ & $\begin{array}{l}\kappa_{1}=C+d\left(\psi^{(0)}(\xi)-\psi^{(0)}(\zeta)+\ln (\zeta-1)-\ln \xi\right) \\
\kappa_{v>1}=\psi_{d}^{(v-1)}(L)+d^{v}\left(\psi^{(v-1)}(\xi)+(-1)^{v} \psi^{(v-1)}(\zeta)\right)\end{array}$ \\
\hline $\mathcal{W}$ & $\begin{array}{l}p(\tau ; \xi, \zeta)=\frac{\Gamma(\zeta)}{\Gamma(\xi) \Gamma(\zeta-\xi)} \frac{\xi}{\zeta}\left(\frac{\xi}{\zeta} \tau\right)^{\xi-1}\left(1-\frac{\xi}{\zeta} \tau\right)^{\zeta-\xi-1} \\
\xi<\zeta, \tau \in\left[0, \frac{\zeta}{\xi}\right]\end{array}$ & $\begin{array}{l}\kappa_{1}=C+d\left(\psi^{(0)}(\xi)-\psi^{(0)}(\zeta)+\ln \zeta-\ln \xi\right) \\
\kappa_{v>1}=\psi_{d}^{(v-1)}(L)+d^{v}\left(\psi^{(v-1)}(\xi)-\psi^{(v-1)}(\zeta)\right)\end{array}$ \\
\hline $\mathcal{M}$ & $\begin{array}{l}p(\tau ; \xi, \zeta)=\frac{\Gamma(\zeta)}{\Gamma(\xi) \Gamma(\zeta-\xi)} \frac{\zeta-1}{\xi-1}\left(\frac{\zeta-1}{\xi-1} \tau\right)^{-\zeta}\left(\frac{\zeta-1}{\xi-1} \tau-1\right)^{\zeta-\xi-1} \\
\xi \leq \zeta, \tau \geq \frac{\xi-1}{\zeta-1}\end{array}$ & $\begin{array}{l}\kappa_{1}=C+d\left(-\psi^{(0)}(\xi)+\psi^{(0)}(\zeta)+\ln (\xi-1)-\ln (\zeta-1)\right) \\
\kappa_{v>1}=\psi_{d}^{(v-1)}(L)+d^{v}(-1)^{v}\left(\psi^{(v-1)}(\xi)-\psi^{(v-1)}(\zeta)\right)\end{array}$ \\
\hline
\end{tabular}

Note: $\psi^{(v)}(L)=\frac{\mathrm{d}^{v+1} \ln \Gamma(L)}{\mathrm{d} L^{v+1}}$ is the ordinary polygamma function, and $\psi_{d}^{(v)}(L)=\sum_{i=0}^{L-1} \psi^{(v)}(L-i)$ is the multivariate extension [9].

By changing the mixing proportions through $w$, the logcumulants of the second order and the third order cover the whole $\kappa_{2}-\kappa_{3}$ plane, which has the same effect as by changing the texture distribution of the product model, as shown in Figure 1a. However, they represent two rather different scattering scenarios, and there is a need to distinguish them.

Denote the log-cumulants of the product model by $\kappa_{v}$ whereas those of the finite mixture model by $\tilde{\kappa}_{v}$ to avoid confusion. When there is an ambiguity between the product model and the finite mixture model, that is $\tilde{\kappa}_{2}=\kappa_{2}$ and $\tilde{\kappa}_{3}=\kappa_{3}$, the difference of the fourth order log-cumulant can be calculated from (18)

$$
f=\tilde{\kappa}_{4}-\kappa_{4}=\frac{k_{3}^{2}-2 k_{2}^{3}-k_{2} k_{4}}{k_{2}} .
$$

Here $k_{v}=\kappa_{v}-\psi_{d}^{(v-1)}(L)$. For all the product models in Table I, it can be shown numerically that $f \leq 0$ holds for all the possible values of distribution parameters. Therefore, if we plot the log-cumulants of the three orders in a 3D space, the result from the finite mixture models will be always below that from the product models, as shown in Figure $1 b$, where the blue surface represents the log-cumulants of the product model and the red and green lines represent examples of mixtures of two components. This $3 \mathrm{D}$ diagram is called log-cumulant cube for simplicity.

To determine whether the product model or the finite mixture model is better for the testing data, a simple approach based on the estimation variance is proposed. By the central limit theorem, the estimation $\hat{\kappa}_{4}$ is Gaussian distributed when the sample size $N$ is large, with variance [15]

$$
\begin{array}{r}
\sigma^{2}=\frac{1}{N}\left[\kappa_{8}+16 \kappa_{2} \kappa_{6}+48 \kappa_{3} \kappa_{5}+34 \kappa_{4}^{2}\right. \\
\left.+72 \kappa_{2}^{2} \kappa_{4}+144 \kappa_{2} \kappa_{3}^{2}+24 \kappa_{2}^{4}\right] .
\end{array}
$$

Therefore, a test statistic to quantitatively evaluate the fit of a product model to the data can be defined as

$$
T=\hat{\kappa}_{4}-\kappa_{4}
$$

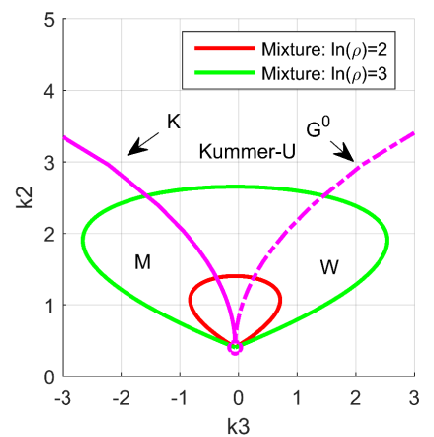

(a)

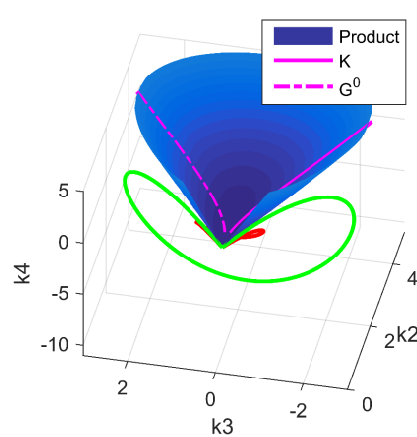

(b)
Fig. 1. The log-cumulant diagram and log-cumulant cube. (a) Log-cumulant diagram. The product models shown in Table I cover the whole diagram. The finite mixture model could also cover the whole diagram by changing the mixing proportions. (b) Log-cumulant cube. Two mixtures (the red line and green line) lie below the blue surface representing the product models.

where $\kappa_{4}$ is calculated from the product model distribution, of which the parameters are estimated using $\hat{\kappa}_{2}$ and $\hat{\kappa}_{3}$ [9]. The absolute value $|T|$ can be viewed as the vertical distance from a point representing the estimated statistics to the product model surface in the log-cumulant cube. If $|T| \leq \sigma$, we can justify that the product model is appropriate for the data, otherwise, the choice of the finite mixture model is preferable.

\section{EXPERIMENTS}

The log-cumulants are tested on both simulated [14] and real SAR data. In the first simulation, we mix two Wishart distributed targets with different proportions. The covariance matrix of the first type is given by (22), which represents an X-Bragg scattering surface with a moderate roughness and a moisture of $40 \%$ [16]. The other covariance matrix is given by (23), representing simple volume scattering. For each covariance matrix, 10000 samples are simulated.

$$
\mathbf{C}_{\text {xbragg }}=\left[\begin{array}{ccc}
1.41 & 0 & 1.89-0.05 j \\
0 & 0.08 & 0 \\
1.89+0.05 j & 0 & 2.57
\end{array}\right]
$$




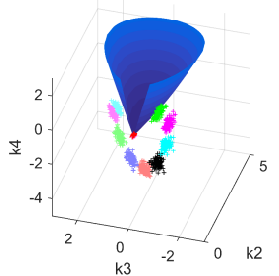

(a)

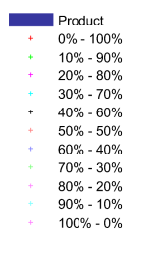

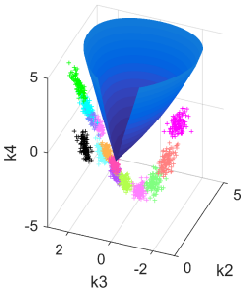

(b)

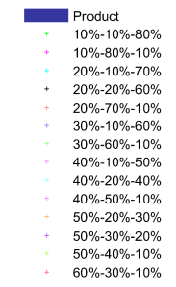

Fig. 2. The log-cumulants of the mixture of 2 components as well as mixture of 3 components. (a) Mixture of 2 targets $\left(\mathbf{C}_{x b r a g g}-\mathbf{C}_{v o l}\right)$. The point clouds representing different mixtures lie below the product model surface. (b) Mixture of 3 targets $\left(\mathbf{C}_{x b r a g g}-\mathbf{C}_{v o l}-\mathbf{C}_{\text {real }}\right)$. Mixture of 3 targets also have $\kappa_{4}$ smaller than the product models that have the same $\kappa_{2}$ and $\kappa_{3}$.

$$
\mathbf{C}_{v o l}=\frac{1}{8}\left[\begin{array}{lll}
3 & 0 & 1 \\
0 & 2 & 0 \\
1 & 0 & 3
\end{array}\right]
$$

Log-cumulants calculated from the simulated data using the bootstrap method [9] are shown in Figure 2a. The result is in agreement with the description of the log-cumulants of the finite mixture model in the previous section, where the sample log-cumulants assume a manifold similar to those in Figure 1b. Mixture of targets can be easily discriminated from the product model by the fourth order log-cumulants $\kappa_{4}$.

Mixtures of three targets are also simulated, with an additional covariance matrix given by (24), which is estimated from a forest area of an AirSAR data acquired over the Netherlands

$$
\mathbf{C}_{\text {real }}=10^{-3} \times\left[\begin{array}{ccc}
161 & -7-4 j & 39-j \\
-7+4 j & 82 & -4+4 j \\
39+j & -4-4 j & 100
\end{array}\right]
$$

Again, the proportions of the mixing components are changed to see their effect on the log-cumulants. It demonstrates again that the mixture of targets have $\tilde{\kappa}_{4}$ smaller than $\kappa_{4}$ of the product model for the same $\kappa_{2}$ and $\kappa_{3}$ as shown in Figure 2b.

The $\kappa_{2}$ and $\kappa_{3}$ are frequently employed to determine the texture distribution of SAR data. However, when the data are very heterogeneous, the use of only the $\kappa_{2}$ and $\kappa_{3}$ is not enough, as the data may be also a mixture of different targets. To show this, a pure target following the Kummer- $\mathcal{U}$ distribution, the mixture of two targets, and the mixture of three targets are simulated, each containing 10000 samples. As shown in Figure 3a, all the simulated data have similar values of $\kappa_{2}$ and $\kappa_{3}$. In this case, wrong conclusions will be drawn from the log-cumulant diagram, as the discrete mixtures will be interpreted as Kummer- $\mathcal{U}$ distributed data. Using the log-cumulant cube, the mixtures are distinguished from the pure target by the $\kappa_{4}$, see Figure $3 \mathrm{~b}$. This is also validated by the quantitative assessment as shown in Table II, where the mixtures have $|T|$ larger than $\sigma$.

The method is also tested on ALOS-2 High-sensitive FullPol mode data, which is acquired over Barcelona (Spain) in March 2015. The original data are in single-look complex format with processing level 1.1 and spatial resolution $3.49 m \times 3.84 m$ (Range $\times$ Azimuth). Before calculating the log-cumulants, a multilook process is applied using a $5 \times 5$

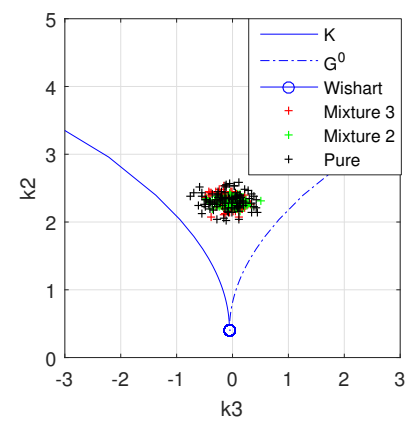

(a)

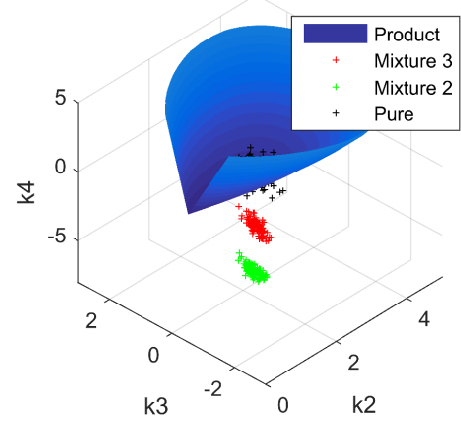

(b)
Fig. 3. The use of $\kappa_{4}$ to distinguish texture from mixture. (a) Log-cumulant diagram. Both textured data and mixtures produce the similar $\kappa_{2}$ and $\kappa_{3}$. (b) Log-cumulant cube. The textured data and mixtures have different $\kappa_{4}$.

TABLE II

Quantitative Assessment on Simulated Data

\begin{tabular}{|l|l|l|l|l|l|}
\hline Test & $\hat{\kappa}_{4}$ & $\kappa_{4}$ & $|T|$ & $\sigma^{2}$ & $\sigma$ \\
\hline \hline Pure & 0.2772 & 0.3910 & 0.1138 & 0.0841 & 0.2900 \\
Mixture 2 & -7.0766 & 0.3863 & $\mathbf{7 . 4 6 2 9}$ & 0.0827 & $\mathbf{0 . 2 8 7 6}$ \\
Mixture 3 & -3.7337 & 0.3982 & $\mathbf{4 . 1 3 1 9}$ & 0.0861 & $\mathbf{0 . 2 9 3 4}$ \\
\hline
\end{tabular}

sliding window. The estimated equivalent number of looks is 14.46. Several regions of interest (ROI) over the urban area and sea area are tested, each covers $20 \times 20$ pixels.

The results are shown in Figure 4. According to the logcumulant diagram, the urban areas could be modeled by different distributions, depending on the composition of the area. For instance, the area with lots of trees (ROI 4-Red) follows a $\mathcal{K}$-distribution, the area with tall apartments (ROI 2-Cyan, 3-Black) follows a Kummer- $U$ distribution, and area with short houses (ROI 1-Blue) can be modeled by a $\mathcal{G}^{0}$ distribution. The sea area is very homogeneous, which can be modeled by the Wishart distribution. However, the logcumulant cube shows that the product model is not appropriate for some urban areas. There are two ROIs (3-Black, 4-Red) producing a $\kappa_{4}$ smaller than 0 , which is far below the surface representing the product model. Two ROIs (1-Blue, 2-Cyan) have part of points above the product model surface as well as part below it, and the quantitative assessment shows that they can be modeled by product models, see Table III. Note that the differences between $|T|$ and $\sigma$ of the ROI 3-Black and ROI 4Red are not so significant as those of the mixtures in Table II, the main reason is that the sample size here is 400 , which gives a larger estimation variance. In order to increase accuracy, additional experiments with more pixels are necessary in the future work.

\section{CONClusions}

Higher order statistics are important to characterize PolSAR data, but there is little knowledge about how to make use of them. In this paper, we demonstrate that the fourth order logcumulant is able to discriminate between different scattering scenarios. In particular, it can be physically interpreted to distinguish the scattering from a single type of target from 


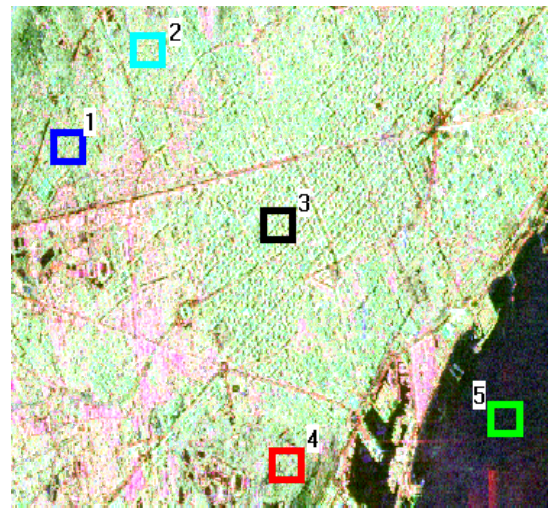

(a)

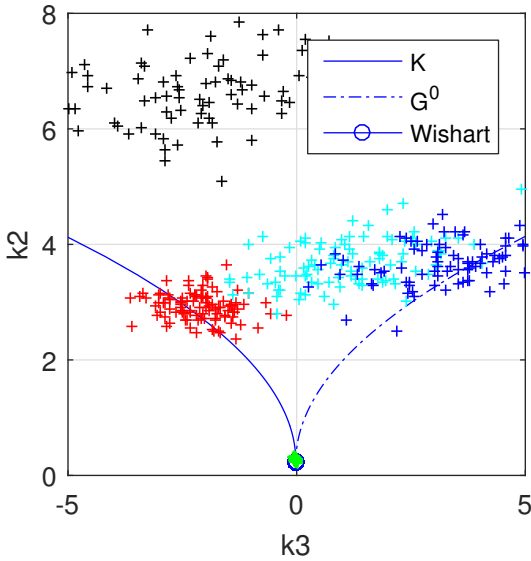

(b)

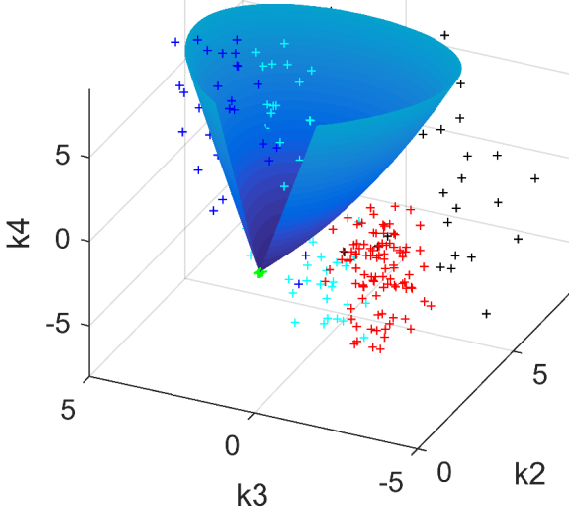

(c)

Fig. 4. The log-cumulants on ALOS2 data. (a) Regions of interest. (b) Log-cumulant diagram. ROIs show different statistics depending on their composition. (c) Log-cumulant cube. It shows some ROIs can be modeled by product models, while others should be represented using finite mixture model as $\kappa_{4}<0$.

TABLE III

QuAntitative Assessment on ALOS-2 DATA

\begin{tabular}{|l|l|l|l|l|l|}
\hline ROI & $\hat{\kappa}_{4}$ & $\kappa_{4}$ & $|T|$ & $\sigma^{2}$ & $\sigma$ \\
\hline \hline 1-Blue & 9.7922 & 9.4141 & 0.3781 & 116.8194 & 10.8083 \\
2-Cyan & 5.0368 & 3.1012 & 1.9356 & 27.0272 & 5.1988 \\
3-Black & -6.3097 & 15.6604 & $\mathbf{2 1 . 9 7 0 1}$ & 376.4956 & $\mathbf{1 9 . 4 0 3 5}$ \\
4-Red & -1.9748 & 3.4908 & $\mathbf{5 . 4 6 5 6}$ & 22.5360 & $\mathbf{4 . 7 4 7 2}$ \\
5-Green & -0.0097 & 0.0028 & 0.0125 & $2.3339 \times 10^{-4}$ & 0.0153 \\
\hline
\end{tabular}

a mixture of targets. As demonstrated both theoretically and experimentally, the mixture of two Wishart distributed components will have a smaller fourth order log-cumulant than the product model distributions with the same second and third order log-cumulants. This result is also extended to other mixtures covering more than two components. As a consequence, it is important to use higher order statistics to texture analysis of PolSAR data, especially when the data are very heterogeneous, as it may result from a mixture of different targets. A diagram combining the second, third and fourth order log-cumulants is suggested to distinguish the product model from finite mixture models, where texture distributions from the Pearson's family are considered. A future work is to test non-Pearson distributions to model the texture, such as the the generalized inverse Gaussian distribution.

\section{ACKNOWLEDGMENT}

The authors would like to thank the Japan Aerospace Exploration Agency (JAXA) for the ALOS-2 data provided in the framework of the 4th ALOS Research Announcement.

\section{REFERENCES}

[1] K. Ward, "Compound representation of high resolution sea clutter," Electronics Letters, vol. 17, no. 16, pp. 561-563, 1981.

[2] J. S. Lee, D. L. Schuler, R. H. Lang, and K. J. Ranson, "K-distribution for multi-look processed polarimetric SAR imagery," in Geoscience and Remote Sensing Symposium, 1994. IGARSS '94. Surface and Atmospheric Remote Sensing: Technologies, Data Analysis and Interpretation., International, vol. 4, (Pasadena, USA), pp. 2179-2181, 1994.
[3] C. C. Freitas, A. C. Frery, and A. H. Correia, "The polarimetric G distribution for SAR data analysis," Environmetrics, vol. 16, no. 1, pp. 13-31, 2005.

[4] L. Bombrun and J.-M. Beaulieu, "Fisher distribution for texture modeling of polarimetric SAR data," IEEE Geoscience and Remote Sensing Letters, vol. 5, no. 3, pp. 512-516, 2008.

[5] L. Bombrun, S. N. Anfinsen, and O. Harant, "A complete coverage of log-cumulant space in terms of distributions for Polarimetric SAR data," in Proc. PolInSAR, (Frascati, Italy), pp. 1-8, 2011.

[6] A. P. Doulgeris, S. N. Anfinsen, and T. Eltoft, "Classification with a nonGaussian model for PolSAR data," IEEE Transactions on Geoscience and Remote Sensing, vol. 46, no. 10, pp. 2999 - 3009, 2008.

[7] Y. Delignon, R. Garello, and A. Hillion, "Etude statistique d'images SAR de la surface de la mer," in 13 Colloque sur le traitement du signal et des images, FRA, 1991, GRETSI, Groupe d'Etudes du Traitement du Signal et des Images, 1991.

[8] J.-M. Nicolas, "Introduction aux statistiques de deuxième espèce: applications des logs-moments et des logs-cumulants à l'analyse des lois d'images radar," TS. Traitement du signal, vol. 19, no. 3, pp. 139-167, 2002.

[9] S. N. Anfinsen and T. Eltoft, "Application of the matrix-variate Mellin transform to analysis of polarimetric radar images," IEEE Transactions on Geoscience and Remote Sensing, vol. 49, no. 6, pp. 2281-2295, 2011.

[10] G. Moser, J. Zerubia, and S. B. Serpico, "Dictionary-based stochastic expectation-maximization for SAR amplitude probability density function estimation," Geoscience and Remote Sensing, IEEE Transactions on, vol. 44, no. 1, pp. 188-200, 2006.

[11] V. Krylov, G. Moser, S. B. Serpico, J. Zerubia, et al., "Modeling the statistics of high resolution SAR images," tech. rep., Institut national de recherche en informatique et en automatique, INRIA, 2008.

[12] Y. Wang, T. L. Ainsworth, and J. Lee, "On characterizing high-resolution SAR imagery using kernel-based mixture speckle models," IEEE Geosci. Remote Sensing Lett., vol. 12, no. 5, pp. 968-972, 2015.

[13] S. N. Anfinsen, "Statistical unimixing of SAR images," tech. rep., Munin Open Research Archive, University of Troms $\varnothing$ The Arctic University of Norway, Troms $\emptyset$, Norway, 4 pp., URL: http://hdl.handle.net/10037/8425, Feb. 2016.

[14] X. Deng, C. López-Martínez, and E. M. Varona, "A physical analysis of polarimetric sar data statistical models," IEEE Transactions on Geoscience and Remote Sensing, vol. PP, no. 99, pp. 1-14, 2016.

[15] S. N. Anfinsen, A. P. Doulgeris, and T. Eltoft, "Goodness-of-fit tests for multilook polarimetric radar data based on the mellin transform," IEEE Transactions on Geoscience and Remote Sensing, vol. 49, no. 7, pp. 2764-2781, 2011.

[16] I. Hajnsek, E. Pottier, and S. R. Cloude, "Inversion of surface parameters from polarimetric SAR," IEEE Transactions on Geoscience and Remote Sensing, vol. 41, no. 4, pp. 727-744, 2003. 